\title{
On Lebesgue Measure of Integral Self-Affine Sets
}

\author{
Ievgen V. Bondarenko • Rostyslav V. Kravchenko
}

Received: 31 March 2010 / Revised: 23 September 2010 / Accepted: 5 October 2010 /

Published online: 23 October 2010

(C) Springer Science+Business Media, LLC 2010

\begin{abstract}
Let $A$ be an expanding integer $n \times n$ matrix and $D$ be a finite subset of $\mathbb{Z}^{n}$. The self-affine set $T=T(A, D)$ is the unique compact set satisfying the equality $A(T)=\bigcup_{d \in D}(T+d)$. We present an effective algorithm to compute the Lebesgue measure of the self-affine set $T$, the measure of the intersection $T \cap(T+u)$ for $u \in \mathbb{Z}^{n}$, and the measure of the intersection of self-affine sets $T\left(A, D_{1}\right) \cap T\left(A, D_{2}\right)$ for different sets $D_{1}, D_{2} \subset \mathbb{Z}^{n}$.
\end{abstract}

Keywords Self-affine set · Tile · Graph-directed system · Self-similar action

Let $A$ be an expanding integer $n \times n$ matrix, where expanding means that every eigenvalue has modulus greater than 1 , and let $D$ be a finite subset of $\mathbb{Z}^{n}$. There exists a unique nonempty compact set $T=T(A, D) \subset \mathbb{R}^{n}$, called (integral) self-affine set, satisfying $A(T)=\bigcup_{d \in D}(T+d)$. It can be given explicitly by

$$
T=\left\{\sum_{k=1}^{\infty} A^{-k} d_{k}: d_{k} \in D\right\} .
$$

The self-affine set $T$ with $|D|=|\operatorname{det} A|$ and of positive Lebesgue measure is called a self-affine tile. Self-affine tiles were intensively studied for the last two decades in the context of self-replicating tilings, radix systems, Haar-type wavelets, etc.

R.V. Kravchenko was partially supported by NSF grant 0503688 .

I.V. Bondarenko $(\bowtie)$

National Taras Shevchenko University of Kiev, Kiev, Ukraine

e-mail: ievgbond@gmail.com

R.V. Kravchenko

Texas A\&M University, College Station, TX, USA 
The question of how to find the Lebesgue measure $\lambda(T)$ of the self-affine set $T$ was considered by Lagarias and Wang in [7], where some partial cases were studied. In particular, it was shown that self-affine tiles have integer Lebesgue measure. He, Lau and Rao [4] reduced the problem of finding $\lambda(T)$ to the case when $D$ is a coset transversal for $\mathbb{Z}^{n} / A\left(\mathbb{Z}^{n}\right)$. The last case was treated by Gabardo and Yu [3] and in more general settings by Bondarenko and Kravchenko [1]. The positivity of the Lebesgue measure of self-affine sets was also studied in $[2,6,8]$.

In this note, we present a simple method to compute the Lebesgue measure $\lambda(T)$ of the self-affine set $T$. We construct a finite labeled graph (automaton) and show that $\lambda(T)$ is equal to the uniform Bernoulli measure of the left-infinite sequences which can be read along paths in this graph. Similar graphs when $D$ is a coset transversal were constructed in $[3,10]$ and other papers. In addition, this method allows finding the measure of the intersection $T \cap(T+u)$ for $u \in \mathbb{Z}^{n}$, and the measure of the intersection of self-affine sets $T\left(A, D_{1}\right) \cap T\left(A, D_{2}\right)$ for different sets $D_{1}, D_{2} \subset \mathbb{Z}^{n}$. Our construction seems to be very natural and actually works for any contracting selfsimilar group action (here the self-affine sets correspond to the self-similar actions of $\mathbb{Z}^{n}$, see [9, Sect. 6.2] and [1]).

We proceed as follows. If the set $D$ does not contain all coset representatives of $\mathbb{Z}^{n} / A\left(\mathbb{Z}^{n}\right)$, we extend it to the set $K \supset D$ which does, and choose a coset transversal $C \subset K$.

We construct a directed labeled graph (automaton) $\Gamma=\Gamma(A, K)$ with the set of vertices $\mathbb{Z}^{n}$, and we put a directed edge from $u$ to $v$ for $u, v \in \mathbb{Z}^{n}$ labeled by the pair $(x, y)$ for $x, y \in K$ if $u+x=y+A v$. The nucleus of the graph $\Gamma$ is the subgraph (subautomaton) $\mathcal{N}$ spanned by all cycles of $\Gamma$ and all vertices that can be reached following directed paths from the cycles. Since the matrix $A$ is expanding, the nucleus $\mathcal{N}$ is a finite graph and it can be algorithmically computed. Indeed, if $u+x=y+A v$ then

$$
\|v\|<\|u\| \quad \text { whenever }\|u\|>\left(1-\left\|A^{-1}\right\|\right)^{-1} \max _{x, y \in K}\left\|A^{-1}(x-y)\right\|,
$$

and the nucleus $\mathcal{N}$ is contained in the ball centered at the origin of radius given by the right-hand side above. Remove every edge in $\mathcal{N}$ whose label is not in $C \times D$, and replace every label $(a, b)$ by $a$. We get some finite graph $\mathcal{N}_{D}$ whose edges are labeled by elements of the set $C$.

Let $C^{-\omega}$ be the space of all left-infinite sequences . . $x_{2} x_{1}, x_{i} \in C$, with the product topology of discrete sets. Let $\mu$ be the uniform Bernoulli measure on $C^{-\omega}$, i.e., the product measure with $\mu(x)=1 /|C|$ for every $x \in C$. For every vertex $v$ of the graph $\mathcal{N}_{D}$, denote by $F_{v}$ the set of all left-infinite sequences which can be read along left-infinite paths in $\mathcal{N}_{D}$ that end in $v$. The sets $F_{v}$ are closed in $C^{-\omega}$, thus compact and measurable.

Theorem 1 The Lebesgue measure of the self-affine set $T$ is equal

$$
\lambda(T)=\sum_{v \in \mathcal{N}_{D}} \mu\left(F_{v}\right) .
$$


Proof Consider the map $\Phi: K^{-\omega} \times \mathbb{Z}^{n} \rightarrow \mathbb{R}^{n}$ given by the rule

$$
\Phi\left(\ldots x_{2} x_{1}, v\right)=v+A^{-1} x_{1}+A^{-2} x_{2}+\cdots,
$$

where $x_{i} \in K$ and $v \in \mathbb{Z}^{n}$. Since $\mathbb{Z}^{n}=K+A\left(\mathbb{Z}^{n}\right)$, the map $\Phi$ is onto (see [8] or [9, Sect. 6.2]). Two elements $\xi=\left(\ldots x_{2} x_{1}, v\right)$ and $\zeta=\left(\ldots y_{2} y_{1}, u\right)$ for $x_{i}, y_{i} \in K$ and $v, u \in \mathbb{Z}^{n}$ represent the same point $\Phi(\xi)=\Phi(\zeta)$ in $\mathbb{R}^{n}$ if and only if there is a finite subset $B \subset \mathbb{Z}^{n}$ and a sequence $\left\{v_{m}\right\}_{m \geq 1} \in B$ such that there exists the path

$$
v_{m} \stackrel{\left(x_{m}, y_{m}\right)}{\longrightarrow} v_{m-1} \stackrel{\left(x_{m-1}, y_{m-1}\right)}{\longrightarrow} \cdots \stackrel{\left(x_{2}, y_{2}\right)}{\longrightarrow} v_{1} \stackrel{\left(x_{1}, y_{1}\right)}{\longrightarrow} u-v
$$

in the graph $\Gamma$ for every $m \geq 1$. Indeed, this path implies that

$$
\begin{gathered}
v_{m}+x_{m}+A x_{m-1}+\cdots+A^{m-1} x_{1}+A^{m} v \\
=y_{m}+A y_{m-1}+\cdots+A^{m-1} y_{1}+A^{m} u .
\end{gathered}
$$

Applying $A^{-m}$ and using the facts that $A^{-1}$ is contracting and the sequence $\left\{v_{m}\right\}_{m \geq 1}$ attains a finite number of values, we get the equality $\Phi(\xi)=\Phi(\zeta)$. For the converse, we choose $v_{m}$ such that (2) holds, and using the equality $\Phi(\xi)=\Phi(\zeta)$ we get that $\left\{v_{m}\right\}_{m \geq 1}$ attains a finite number of values. Notice that since the set $B$ is assumed to be finite, every element $v_{m}$ lies either on a cycle or there is a directed path from a cycle to $v_{m}$. In particular, all elements $v_{m}$ should belong to the nucleus $\mathcal{N}$, and we have that the elements $\xi$ and $\zeta$ represent the same point in $\mathbb{R}^{n}$ if and only if there exists a left-infinite path in $\mathcal{N}$ labeled by $\left(\ldots x_{2} x_{1}, \ldots y_{2} y_{1}\right)$ and ending in $u-v$.

Take the restriction $\Phi_{C}: C^{-\omega} \times \mathbb{Z}^{n} \rightarrow \mathbb{R}^{n}$ of the map $\Phi$ on the subset $C^{-\omega} \times \mathbb{Z}^{n}$. Since $\mathbb{Z}^{n}=C+A\left(\mathbb{Z}^{n}\right)$, the map $\Phi_{C}$ is also onto, and this gives an encoding of points in $\mathbb{R}^{n}$ by elements of $C^{-\omega} \times \mathbb{Z}^{n}$. Consider the uniform Bernoulli measure $\mu$ on the space $C^{-\omega}$ and the counting measure on the group $\mathbb{Z}^{n}$, and put the product measure on the space $C^{-\omega} \times \mathbb{Z}^{n}$. Since the set $C$ is a coset transversal, the push-forward of this measure under $\Phi_{C}$ is the Lebesgue measure on $\mathbb{R}^{n}$ (see [1, Proposition 25]). Hence to find the Lebesgue measure of the self-affine set $T$ it is sufficient to find the measure of its preimage in $C^{-\omega} \times \mathbb{Z}^{n}$. However, $T$ is equal to $\Phi\left(D^{-\omega} \times 0\right)$, and hence the sequence $\left(\ldots x_{2} x_{1}, v\right)$ for $x_{i} \in C$ and $v \in \mathbb{Z}^{n}$ represents a point in $T$ if and only if there exists a left-infinite path in the nucleus $\mathcal{N}$, which ends in $-v$ and is labeled by $\left(\ldots x_{2} x_{1}, \ldots y_{2} y_{1}\right)$ for some $y_{i} \in D$. Hence

$$
\Phi_{C}^{-1}\left(\Phi\left(D^{-\omega} \times 0\right)\right)=\bigcup_{v \in \mathcal{N}_{D}} F_{v} \times\{-v\},
$$

and the statement follows.

The Bernoulli measure of the sets $F_{v}$ for any finite graph $\Gamma=(V, E)$ can be effectively computed (see [1, Sect. 2]). First, we can assume that the graph is leftresolving, i.e., for every vertex $v \in V$ the incoming edges to $v$ have different labels. Indeed, for any finite graph $\Gamma=(V, E)$ there exists a left-resolving graph $\Gamma^{\prime}=\left(V^{\prime}, E^{\prime}\right)$ with the property that for every $v \in V$ there exists $v^{\prime} \in V^{\prime}$ such 


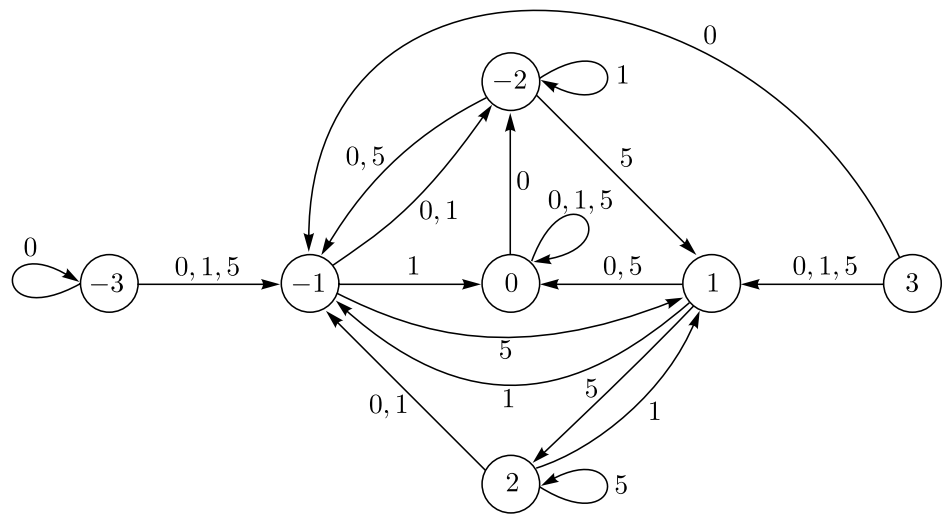

Fig. 1 The graph $\mathcal{N}_{D}$ for $A=(3)$ and $D=\{0,1,5,6\}$

that $F_{v}=F_{v^{\prime}}$, and this graph can be easily constructed (here every vertex $v^{\prime}$ corresponds to some subset of $V$, see [5, Sect. 2.3]). For a left-resolving graph, the vector $\left(\mu\left(F_{v}\right)\right)_{v \in V}$ (if it is nonzero) is the left eigenvector of the adjacency matrix of the graph for the eigenvalue $|C|=|\operatorname{det} A|$. This eigenvector is uniquely defined if we know its entries $\mu\left(F_{v}\right)$ for vertices $v$ in the strongly connected components without incoming edges. For every such a component $\Gamma^{\prime}$, we have $F_{v}=C^{-\omega}$ and $\mu\left(F_{v}\right)=1$ for every vertex $v$ in $\Gamma^{\prime}$ if inside this component every vertex has incoming edges labeled by every element of the set $C$, and $\mu\left(F_{v}\right)=0$ otherwise. In particular, the entries $\mu\left(F_{v}\right)$ are rational numbers, and we recover the following result of [4].

\section{Corollary 2 Every self-affine set has rational Lebesgue measure.}

It is also easy to check when the measure of $T$ is non-zero without calculating its precise value but just looking at the left-resolving graph (not the graph $\mathcal{N}_{D}$ ) constructed above. The measure $\lambda(T)$ will be positive if and only if there exists a strongly connected component such that inside this component every vertex has incoming edges labeled by every letter of the alphabet.

Example 1 Let $A=(3)$ and $D=\{0,1,5,6\}$. The self-affine set $T$ is $\left[0, \frac{4}{3}\right] \cup\left[\frac{5}{3}, 3\right]$, and $\lambda(T)=8 / 3$. Choose $K=D$ and the coset transversal $C=\{0,1,5\}$. The associated automaton $\mathcal{N}_{D}$ is shown in Fig. 1 . Here $\mu\left(F_{0}\right)=1, \mu\left(F_{1}\right)=1 / 3, \mu\left(F_{2}\right)=1 / 8$, $\mu\left(F_{-1}\right)=7 / 12, \mu\left(F_{-2}\right)=5 / 8$, and $\mu\left(F_{-3}\right)=\mu\left(F_{3}\right)=0$.

The above method can be used to find $\lambda(T \cap(T+u))$ for $u \in \mathbb{Z}^{n}$. The set $T+u$ is the image of the set $D^{-\omega} \times u$, and its preimage under $\Phi_{C}$ can be described as in (3). In particular,

$$
\lambda(T \cap(T+u))=\sum_{\substack{v_{1}, v_{2} \in \mathcal{N}_{D} \\ u=v_{2}-v_{1}}} \mu\left(F_{v_{1}} \cap F_{v_{2}}\right) .
$$


Similarly, one can find the measure of the intersection of self-affine sets $T_{1}=$ $T\left(A, D_{1}\right)$ and $T_{2}=T\left(A, D_{2}\right)$ for different sets $D_{1}, D_{2} \subset \mathbb{Z}^{n}$. We take a set $E$ which contains $D_{1}, D_{2}$, and some coset transversal $C$, and as above we construct the nucleus $\mathcal{N}$ and its subgraphs $\mathcal{N}_{D_{1}}$ and $\mathcal{N}_{D_{2}}$. Then

$$
\lambda\left(T_{1} \cap T_{2}\right)=\sum_{v \in \mathcal{N}} \mu\left(F_{v}^{(1)} \cap F_{v}^{(2)}\right),
$$

where $F_{v}^{(i)}$ is calculated in the graph $\mathcal{N}_{D_{i}}$. Hence these two problems are reduced to the question of how to find the measure of the intersection $F_{v_{1}}^{(1)} \cap F_{v_{2}}^{(2)}$, where each set $F_{v_{i}}^{(i)}$ is defined in some finite graph $\Gamma^{(i)}=\left(V^{(i)}, E^{(i)}\right)$ with its vertex $v_{i}$. One can construct a new finite graph $\Gamma$ (sometimes called the labeled product of graphs $\Gamma^{(i)}$ ) with the set of vertices $V^{(1)} \times V^{(2)}$, where we put an edge $\left(u_{1}, u_{2}\right) \stackrel{x}{\rightarrow}\left(w_{1}, w_{2}\right)$ for every edges $u_{1} \stackrel{x}{\rightarrow} w_{1}$ in $\Gamma^{(1)}$ and $u_{2} \stackrel{x}{\rightarrow} w_{2}$ in $\Gamma^{(2)}$. Then $F_{\left(v_{1}, v_{2}\right)}=F_{v_{1}}^{(1)} \cap F_{v_{2}}^{(2)}$ (see [5, Sect. 3.2]).

\section{References}

1. Bondarenko, I., Kravchenko, R.: Graph-directed systems and self-similar measures on limit spaces of self-similar groups. Adv. Math. (2010). doi:10.1016/j.aim.2010.09.018. Available at arXiv:1001.2291 [abs]

2. Deng, G.-T., He, X.-G.: Integral self-affine sets with positive Lebesgue measures. Arch. Math. 90(2), 150-157 (2008)

3. Gabardo, J.-P., Yu, X.: Natural tiling, lattice tiling and Lebesgue measure of integral self-affine tiles. J. Lond. Math. Soc. II 74(1), 184-204 (2006)

4. He, X.-G., Lau, K.-S., Rao, H.: Self-affine sets and graph-directed systems. Constr. Approx. 19(3), 373-397 (2003)

5. Hopcroft, J.E., Ullman, J.D.: Introduction to Automata Theory, Languages and Computation. Addison-Wesley, Reading (1979)

6. Kirat, I.: On the Lebesgue measure of self-affine sets. Turk. J. Math. 25(4), 535-543 (2001)

7. Lagarias, J.C., Wang, Y.: Integral self-affine tiles in $\mathbb{R}^{n}$. I: Standard and nonstandard digit sets. J. Lond. Math. Soc. II 54(1), 161-179 (1996)

8. Lagarias, J.C., Wang, Y.: Self-affine tiles in $\mathbb{R}^{n}$. Adv. Math. 121(1), 21-49 (1996)

9. Nekrashevych, V.: Self-Similar Groups. Mathematical Surveys and Monographs, vol. 117. American Mathematical Society, Providence (2005)

10. Scheicher, K., Thuswaldner, J.M.: Canonical number systems, counting automata and fractals. Math. Proc. Camb. Philos. Soc. 133(1), 163-182 (2002) 